\title{
Induction of c-Myc-dependent cell proliferation through toll-like receptor 3 in head and neck cancer
}

\author{
RALPH PRIES $^{1 *}$, LISA HOGREFE ${ }^{1 *}$, LEI XIE $^{2}$, HENNING FRENZEL $^{1}$, \\ CARSTEN BROCKS $^{1}$, CLAUDIA DITZ ${ }^{1}$ and BARBARA WOLLENBERG ${ }^{1}$ \\ ${ }^{1}$ Department of Otorhinolaryngology, University of Schleswig-Holstein, Campus Lübeck, D-23538 Lübeck, Germany; \\ ${ }^{2}$ Department of Head and Neck Surgery, Sir Run Run Shaw Hospital, Zhejiang University, 310016 Hangzhou, P.R. China
}

Received August 22, 2007; Accepted October 2, 2007

\begin{abstract}
Protein expression of human toll-like receptors (TLR) 1-10 was measured in cell lines and solid tumors of head and neck squamous cell carcinoma (HNSCC). All HNSCC cell lines and $80 \%$ of solid tumors were found to express TLR3 as a predominantly intracellular protein, while no other TLR proteins were expressed. TLR3 has previously been shown to contribute to the activation of nuclear factor- $\mathrm{kB}$ (NF-кB), a transcription factor which promotes several types of human cancers. Significantly, NF- $\kappa \mathrm{B}$ expression was strongest in protein extracts from carcinoma tissue in which TLR3 was overexpressed. Inhibition of TLR3 expression in permanent HNSCC cell lines resulted in decreased expression of the oncoprotein c-Myc resulting in decreased cell proliferation. Correspondingly, overexpression of human TLR3 in mouse fibroblasts resulted in an upregulation of c-Myc and increased sensitivity for PolyI:C-induced cell proliferation. Our data suggest that TLR3 contributes to the malignant phenotype leading to invasive carcinoma in HNSCC.
\end{abstract}

\section{Introduction}

The innate immune system builds up the host defence against a huge diversity of pathogens like viruses, bacteria and fungi. They are recognized by so called pattern recognition receptors (PRRs), such as the toll-like receptors (TLRs), which trigger the initiation of various defence mechanisms.

In humans 10 different TLRs have been described which are expressed on various immune cells and a variety of stromal cells. The expression of TLRs represents an important link between innate and adaptive immune responses (1) and a set of various transcription factors is known to be activated via TLRdependent mechanisms (2). This complex network requires a

Correspondence to: Dr Barbara Wollenberg, UK-SH, Klinik für HNO, Ratzeburger Allee 160, D-23538 Lübeck, Germany

E-mail: barbara.wollenberg@hno.uni-luebeck.de

*Contributed equally

Key words: tumor immunity, toll-like receptor 3, head and neck squamous cell carcinoma, nuclear factor $\mathrm{\kappa B}, \mathrm{c}-\mathrm{Myc}$ well-tuned regulation to ensure physiologic cell growth since dysregulation of transcription factors such as nuclear factor $\mathrm{\kappa B}$ $(\mathrm{NF}-\mathrm{\kappa B})$ participate in many distinct aspects of oncogenesis (3).

TLR3 was first cloned from a human placenta-derived cDNA library and encodes a protein with a calculated weight of $97 \mathrm{kDa}$ (4). Double-stranded RNA (dsRNA) is known to be recognized by a homodimer of TLR3 which leads to the activation of NF-кB $(5,6)$. This transcriptional activator is able to suppress apoptosis and can induce the expression of proto-oncogenes such as c-myc and various additional genes, all of which promote tumor cell invasion and angiogenesis (7) in cancers, including head and neck cancer (head and neck squamous cell carcinoma, HNSCC) (3).

Head and neck squamous cell carcinoma (HNSCC) is the sixth most common tumor occurring almost exclusively among middle-aged tobacco and alcohol abusers (8). Cells of head and neck cancer are known to develop molecular strategies to escape efficient antitumor immune responses. It is supposed that tumor production of various immunosuppressive mediators contributes to suppressed immune functions. The molecular mechanisms responsible for these malignant transformation processes and the development of the immunosuppressive HNSCC microenvironment remain mostly unknown (9-15).

In this study we demonstrated increased expression levels of NF- $\mathrm{kB}$ with HNSCC tumor progression and presented the first evidence that TLR3 is strongly expressed in cells of human HNSCC. The expression of TLR3 was detected only in malignant cells and was clearly associated with high protein levels and activity of the transcriptional activator NF$\kappa \mathrm{B}$. Inhibition of the predominantly intracellular protein by TLR3-specific siRNA resulted in decreased expression of cMyc and decreased cell proliferation. Overexpression of TLR3 in mouse fibroblasts led to an upregulation of c-Myc and an increased sensitivity for PolyI:C-induced cell proliferation in these cells. Thus our data strongly suggest a significant role of TLR3 during the progression of head and neck cancer by triggering $\mathrm{NF}-\kappa \mathrm{B}-d e p e n d e n t$ signal transduction pathways.

\section{Materials and methods}

Human tissue samples and HNSCC cell lines. After written informed consent, tumor tissue specimens were obtained 
during standard surgical procedure. Simultaneously tumordraining lymph nodes were obtained by neck dissection and examined by a pathologist. Specimens of tonsils were obtained from children undergoing surgery for tonsillar hyperplasia, and healthy nasal mucosa was obtained from patients undergoing functional surgery of the nasal septum. Specimens of normal oropharyngeal mucosa were obtained from patients with sleep-related breathing disorders undergoing uvulopalatopharyngoplasty. Tissue specimens were transported in sterile saline and processed immediately after excision. The use of human tissues for research purposes was approved by the ethics committee of the University of Lübeck.

Permanent HNSCC cell lines from different anatomical sites were used, such as human pharynx (ANT-1, FADU), tongue (CAL27), larynx (PCI1, HLac79), and oral cavity (PCI13). Protein expression was measured for TLR1-10 in 30 different HNSCC tissue samples as well as in 8 different HNSCC cell lines. HNSCC cell lines PCI1 and BHY were transfected with TLR3 siRNA in a standardized procedure with Lipofectamine 2000 (InvivoGen). Transfection efficiency was investigated using the BLOCK-iT-Fluorescent Oligo (Invitrogen).

Mouse fibroblasts NIH3T3 were used for transfection with plasmid pUNO-hTLR3 (Invitrogen). This plasmid encodes for human TLR3 driven from a strong hEF1-HTLV promoter. The plasmid encodes the $b s r$ (blasticidin resistance gene) from Bacillus cereus that confers resistance to the antibiotic Blasticidin $\mathrm{S}$ and allows blasticidin selection in both mammalian cells and $E$. coli bacteria.

Specific inhibition of c-Myc was achieved using the Int-H1-S6A, F8A c-Myc inhibitor peptide. Control for this peptide is $\mathrm{H} 1-\mathrm{S} 6 \mathrm{~A}, \mathrm{~F} 8 \mathrm{~A}$, which lacks the N-terminal internalization sequence [Biomol, Hamburg; (16)]. NF-кB activity was analyzed using the reporter plasmid pNF-кBhrGFP (Stratagene) that confers resistance to the antibiotic hygromycin.

Western blot analysis. Cell extracts were prepared and solubilized and protein concentrations were determined using the Bio-Rad protein assay kit (Bio-Rad GmbH, Munich, Germany) with bovine serum albumin (BSA) as a standard. Aliquots of protein extracts $(20 \mu \mathrm{g})$ were run on $10 \%$ acrylamide SDS-PAGE and were then transferred to a nitrocellulose membrane. Blots were decorated with specific antibodies (Biomol, Hamburg, Germany) as specified in the figure legends.

Flow cytometry. Surface antigen staining was performed as described previously (17) and for intracellular staining saponin (Sigma-Aldrich) was used for the permeabilization of cell membranes. The PE (phycoerthrin)-labelled anti-human TLR3 antibody was purchased from eBioscience. Samples were analyzed on a FACSCanto (Becton Dickinson). Data acquisition and analysis were performed using FACSDiva software (Becton Dickinson).

MTT assay. For the quantitative determination of cellular proliferation an MTT assay (Promega) was used, which is based on the cleavage of the yellow tetrazolium salt MTT into purple formazan by metabolically active cells. The solubilized formazan product can be photometrically quantitated using an ELISA reader. An increase in the number of living cells results in an increase in total metabolic activity which leads to a stronger colour formation. Experiments were performed at least three times for each data point.

\section{Results}

TLR3 is a predominantly intracellular protein in HNSCC. It is generally accepted that leucine-rich repeats of TLRs signal the presence of microbial patterns and activate various transcription factors like NF- $\mathrm{KB}$, some of which are known to participate in different kinds of oncogenesis.

We investigated the expression levels of human TLR proteins in various human HNSCC cell lines. Western blot experiments revealed a strong expression of TLR3 protein in all analyzed HNSCC cell lines (Fig. 1A), whereas no other TLR proteins (1-2 and 4-10) could be detected. The transcriptional activator protein $\mathrm{NF}-\kappa \mathrm{B}$ was additionally measured and found to be equally strongly expressed in all the HNSCC cell lines that were analyzed (Fig. 1A). The subcellular localization of TLR3 in cells of HNSCC was analyzed by flow cytometry. Permanent HNSCC cell lines PCI1 and PCI13 were used for flow cytometric analysis using an anti-TLR3-PE antibody, whereas intracellular staining of TLR3 was carried out using saponin for permeabilization of cell membranes. Our data indicate a predominantly intracellular localization of TLR3 in cells of HNSCC and a weak surface expression (Fig. 1B).

TLR expression levels were examined in primary human HNSCC tumors from various sites in the head and neck region (tonsil, larynx, tongue, hypopharynx and the locoregional-draining lymph nodes). Western blot analysis revealed a strong expression of TLR3 and NF- $\mathrm{\kappa B}$ in $~ 80 \%$ of the analyzed tumors and lymph node metastases (Fig. 1A), whereas 30 independent tissue samples were analyzed. Thus, our data represent the first identification of TLR3 expression in cells of human head and neck squamous cell carcinoma and strongly suggest a link between TLR3 and NF- $\mathrm{KB}$ in HNSCC.

TLR3 expression is associated with malignant transformed epithelial tissue. To elucidate the connection between TLR3 and $\mathrm{NF}-\mathrm{KB}$ during the progression of oncogenesis in head and neck squamous cell carcinoma, HNSCC was compared with several other tissue samples. Mucosal tissue from the soft palate and the anterior tonsillar arch of healthy patients served as the primary control. No significant levels of TLR3 or NF- $\mathrm{KB}$ protein could be detected in these samples (data not shown).

To determine a possible involvement of TLR3 and NF-кB during the initiating steps in the development of a malignant lesion, we investigated the expression of TLR3 and NF-KB in the oral mucosa of three heavily smoking healthy volunteers without HNSCC. Due to the underlying chronic inflammation $\mathrm{NF}-\kappa \mathrm{B}$ was strongly expressed in all three samples, whereas no TLR3 protein could be detected (Fig. 1A).

Squamous cell carcinoma develops through a series of steps such as dysplastic tissue alterations found adjacent to the carcinoma or leukoplasia as a progenitor of the malignant 
A

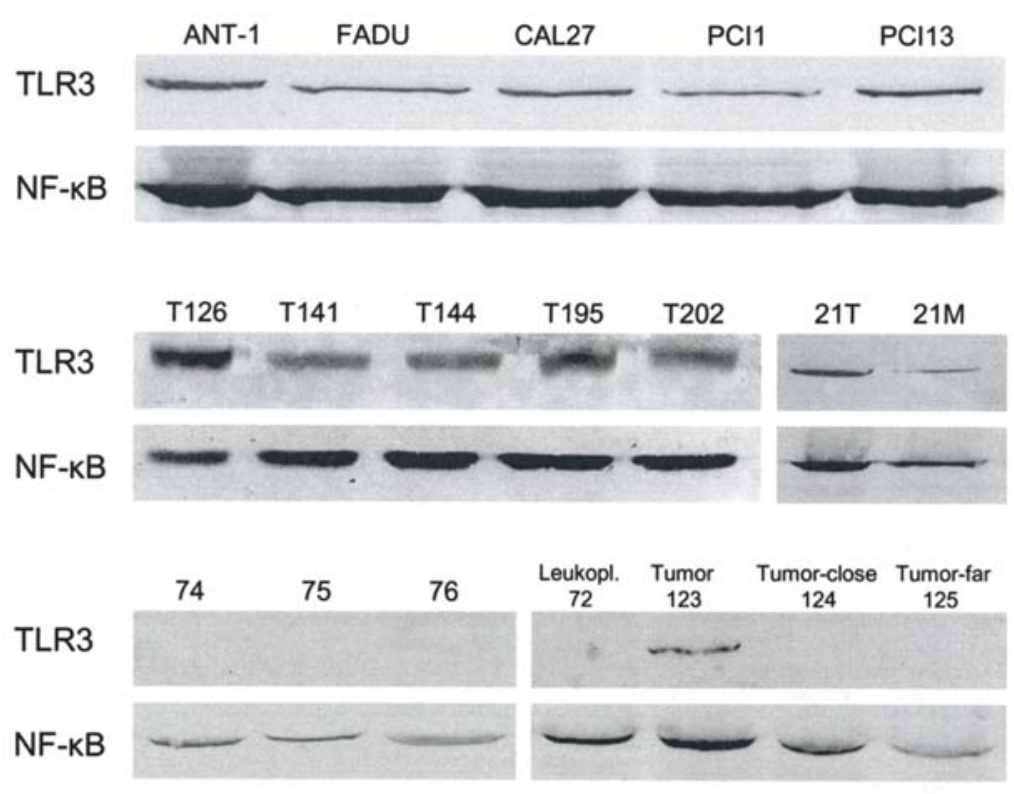

B
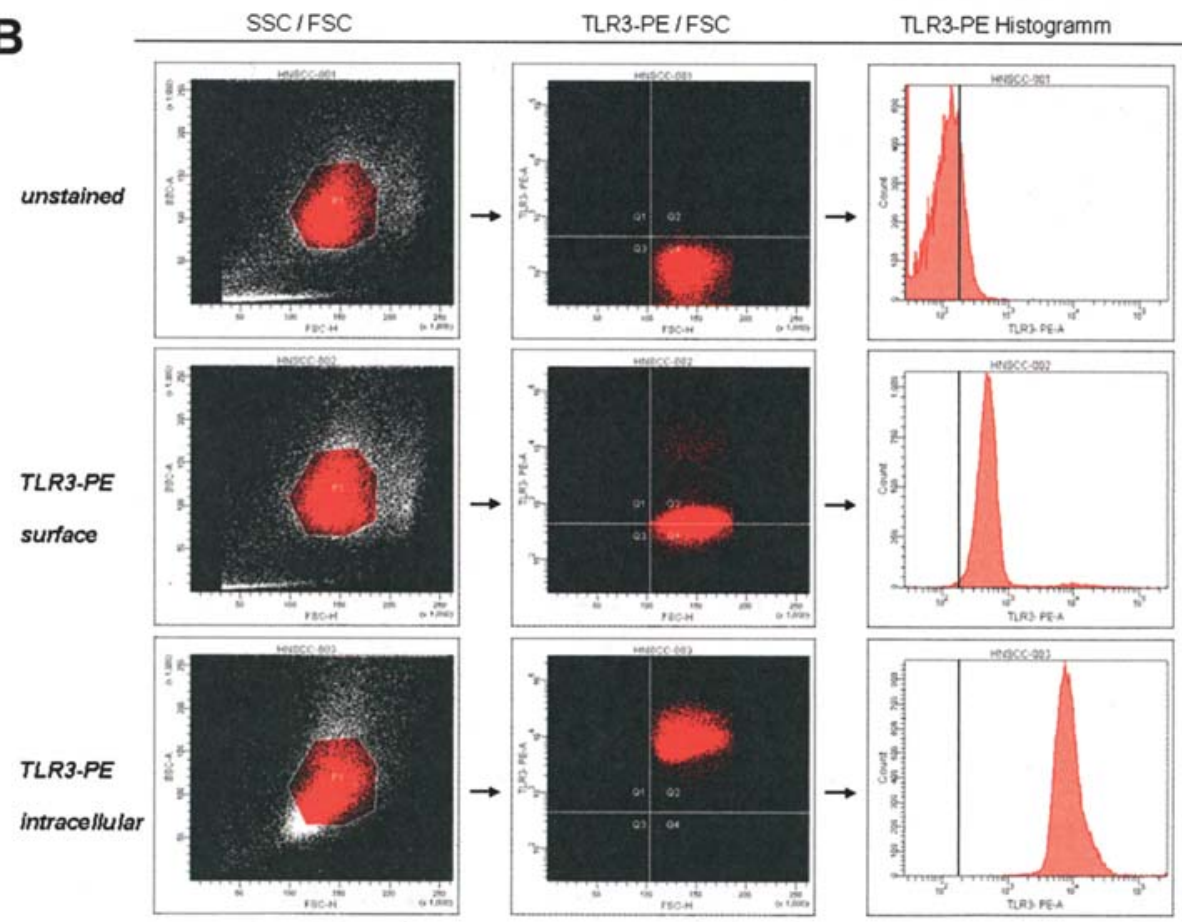

TLR3-PE Histogramm

Figure 1. TLR3 is expressed as a predominantly cytoplasmic protein in cells of HNSCC. (A) Expression levels of TLR3 and NF- $\mathrm{B}$ in various HNSCC cell lines. Crude protein extracts were prepared from the HNSCC cell lines ANT-1, FADU, and PCI1. Tissue samples from a hypopharynx carcinoma (T126), a laryngeal carcinoma (T141, T144), a tonsil carcinoma (T195, T202), a hypopharyngeal-laryngeal carcinoma (sample 21T), and metastatic tissue from the

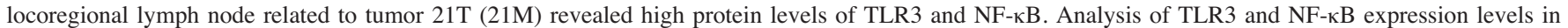
protein extracts prepared from the oral mucosa of three heavy smoking patients without HNSCC (samples 74-76) and the neighbouring tissues of a solid hypopharynx-larynx carcinoma (123) at a 5-mm (tumor-close, 124) distance and a 10-cm (tumor-far, 125) distance from the tumor and leukoplastic tissue (72). (B) Flow cytometric analysis using a TLR3-PE antibody revealed that TLR3 is a predominantly cytoplasmic protein and only weakly expressed on the cell surface.

lesion. Therefore, protein extracts were prepared from the neighbouring tissues of a solid hypopharynx-larynx carcinoma at a 5-mm (tumor-close) distance and a $10-\mathrm{cm}$ (tumor-far) distance from the tumor and leukoplastic tissue. High TLR3 levels were detected in tumor tissue, but not in tissue $10 \mathrm{~cm}$ adjacent to the tumor or even at $5 \mathrm{~mm}$ adjacent to the tumor. TLR3 expression was also undetectable in leukoplastic tissue
(Fig. 1A). NF-кB was expressed in all analyzed tissues due to an underlying inflammatory microenvironment, whereas the highest levels of NF- $\mathrm{BB}$ protein were found in the hypopharyngeal-laryngeal carcinoma. Significantly lower protein levels were found in the leukoplastic tissue and even lower levels in the analyzed samples taken from tissue next to the tumor (Fig. 1A). These data indicate an increasing 
A

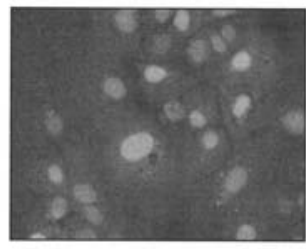

B

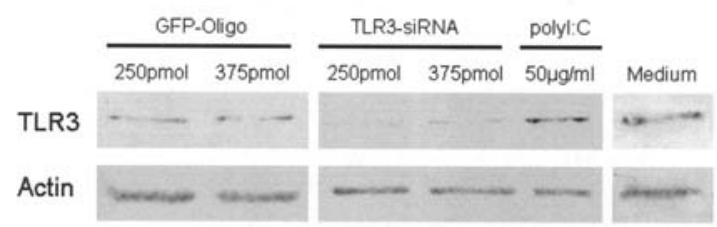

C

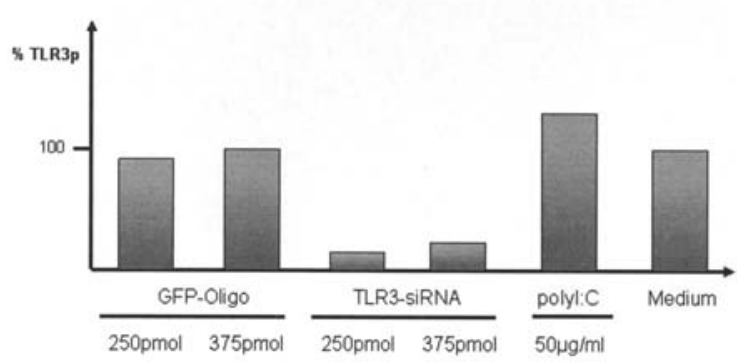

Figure 2. siRNA mediated inhibition of TLR3. (A) GFP-labelled control oligonucleotides were used to analyse transfection efficiencies by fluorescence microscopy, indicating transfection efficiencies always $>80 \%$. (B) Western blot analysis revealed a significant decrease in TLR3 expression in response to TLR3 siRNA transfection, whereas cells grown in the presence of $50 \mu \mathrm{g} / \mathrm{ml}$ polyI:C showed a slightly increased expression of TLR3 compared to the control medium. Experiments were carried out with two different HNSCC cell lines leading to equal results (C).

expression of the transcriptional activator $\mathrm{NF}-\kappa \mathrm{B}$ during the progression of HNSCC tumorigenesis. High levels of TLR3 protein were exclusively detected in cells of HNSCC and were highly correlated with a strong NF- $\mathrm{KB}$ expression.

TLR3 inhibition affects c-Myc expression and cell proliferation. Specific siRNA was transfected in two different HNSCC cell lines to inhibit the expression of TLR3. Various siRNA and Lipofectamine concentrations were tested using fluorescent-labelled control siRNA. Optimized parameters were identified by fluorescence microscopy and flow cytometry and revealed transfection efficiencies always $>80 \%$ (Fig. 2A).

Protein analyses indicated a strong and specific inhibition of TLR3 expression in siRNA-transfected cells, whereas cells transfected with green fluorescent protein (GFP)labelled control oligonucleotides revealed a TLR3 expression similar to non-transfected cells (Fig. 2B). Cells grown in the presence of the TLR3 ligand PolyI:C, which resembles artificial double-stranded RNA, showed a slightly increased expression of TLR3 protein (Fig. 2B).

Expression analysis of various HNSCC-related proteins such as $\mathrm{NF}-\kappa \mathrm{B}$, cyclin D1, or p53 revealed no effects in response to an inhibition of TLR3 (Fig. 3A).

Noteworthy, expression of onco-protein c-Myc was significantly decreased in TLR3 siRNA transfected cells of HNSCC (Fig. 3A). Since c-Myc is known to be involved in
A

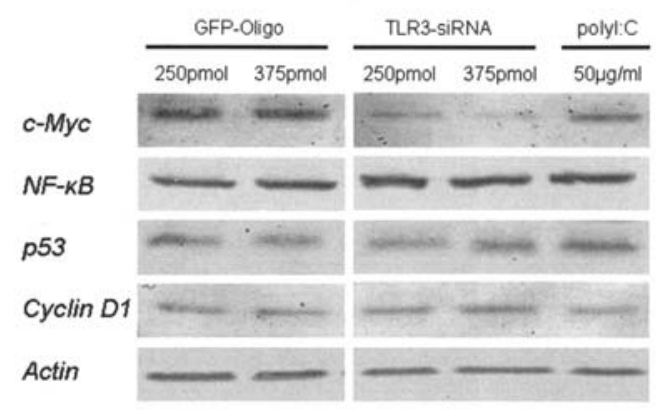

B

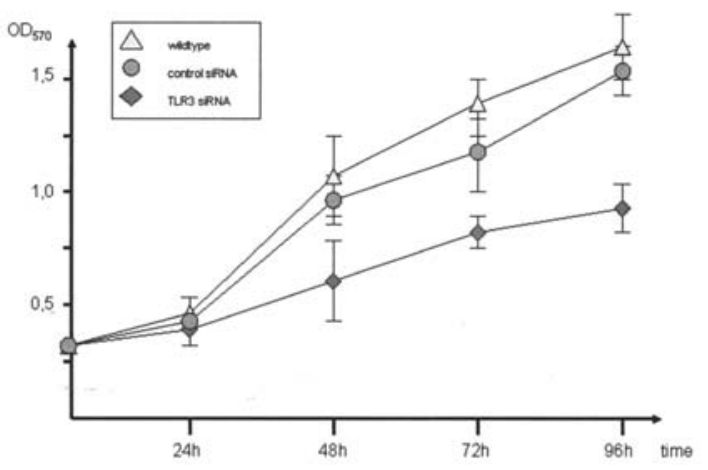

Figure 3. Inhibition of TLR3 affects c-Myc and cell growth. (A) Protein extracts of cells transfected with GFP-labelled control oligonucleotides, TLR3 siRNA or grown in the presence of polyI:C were analysed with respect to the protein levels of $\mathrm{c}-\mathrm{Myc}, \mathrm{NF}-\mathrm{\kappa B}, \mathrm{p} 53$, and cyclin D1. Actin was used as a loading control. (B) Inhibition of TLR3 results in affected cell growth. Growth analysis of HNSCC cells transfected either with TLR3 siRNA or GFP-labelled control oligonucleotides compared with nontransfected cells (wild-type). Experiments were performed at least three times for each data point with deviations $<10 \%$.

cell cycle regulation and cell proliferation, we analyzed cell growth of HNSCC cells transfected either with TLR3 siRNA or GFP-labelled control oligonucleotides as well as of nontransfected cells. Cell growth was investigated photometrically for $96 \mathrm{~h}$ using an MTT assay. Fig. 3B clearly illustrates decreased cell growth of TLR3 siRNA-transfected cells whereas growth of cells transfected with GFP-labelled control oligonucleotides was not significantly affected compared to the wild-type situation.

TLR3 overexpression triggers $c-M y c$ in murine fibroblasts. To verify that TLR3 regulates c-Myc expression and to exclude non-specific effects of siRNA as previously described (18), we overexpressed human TLR3 in murine NIH3T3 fibroblasts and monitored c-Myc expression and cell proliferation. NIH3T3 fibroblasts were transfected with a plasmid (pUNOhTLR3) encoding for human TLR3 driven from a strong hEF1-HTLV promoter.

NIH3T3 fibroblasts were recently shown to express TLR3 and are thus able to propagate this toll-like receptor. Since transfected human TLR3 protein has a different molecular weight from the endogenous murine TLR3 protein, expression analyses of the respective proteins by Western hybridization were able to be performed. Cells of HNSCC revealed significantly higher TLR3 protein levels compared to wild-type NIH3T3 fibroblasts (Fig. 4A). Fig. 4 illustrates the additional 
A

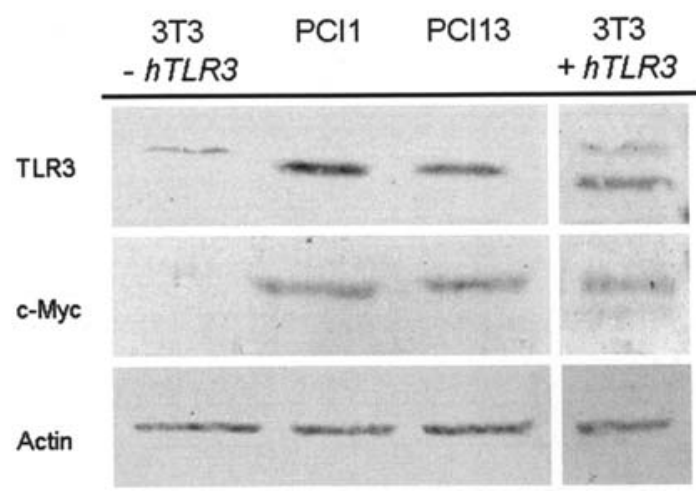

B
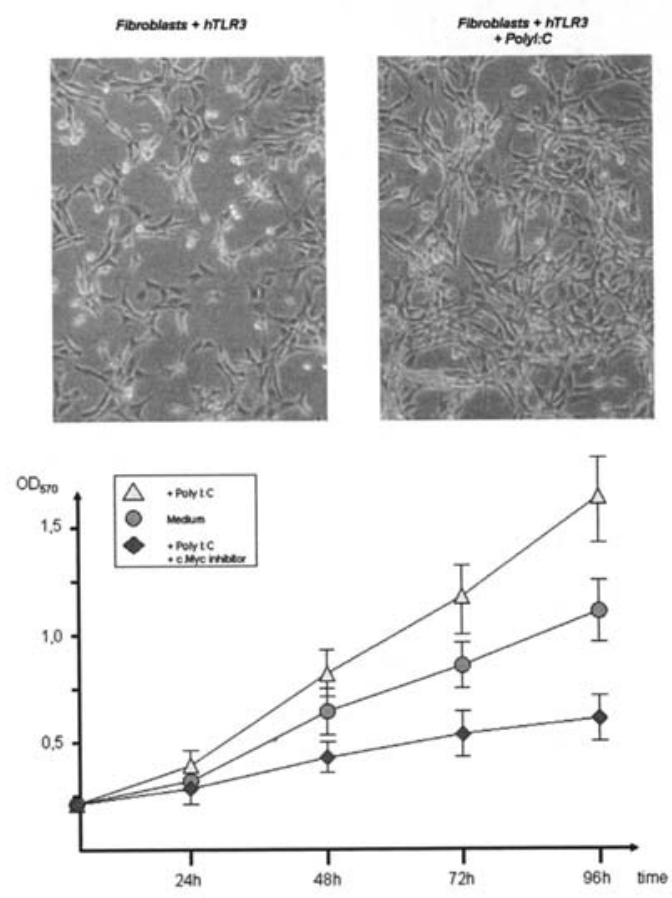

Figure 4. Expression of human TLR3 in NIH3T3 fibroblasts. (A) Heterologous expression of human TLR3 in NIH3T3 fibroblasts resulted in an increased expression of c-Myc. (B) Mouse NIH3T3 fibroblasts expressing human TLR3 revealed an increased sensitivity to PolyI:C- induced cell proliferation as illustrated by microscopy and MTT cell proliferation assays in the presence and absence of the TLR3 ligand PolyI:C. Inhibition of cMyc function using the c-Myc inhibitory peptide Int-H1-S6A impaired the growth-stimulating effect of PolyI:C.

expression of human TLR3 in transfected NIH3T3 fibroblasts resulting in an expression of c-Myc protein in these cells, which could not be detected in untransfected NIH3T3 fibroblasts (Fig. 4A).

Heterologous expression of human TLR3 in NIH3T3 fibroblasts resulted furthermore in increased cell growth in response to the TLR3 ligand PolyI:C. Inhibition of c-Myc function using the c-Myc inhibitory peptide Int-H1-S6A impaired the growth-stimulating effect of PolyI:C (Fig. 4B). These data establish a role of TLR3 in the regulation of cMyc expression and HNSCC cell proliferation.

TLR3 stimulation triggers $N F-\kappa B$ activation. To verify the link between TLR3 stimulation and NF- $\mathrm{B}$ activity, NIH3T3 fibroblasts were simultaneously transfected with TLR3-

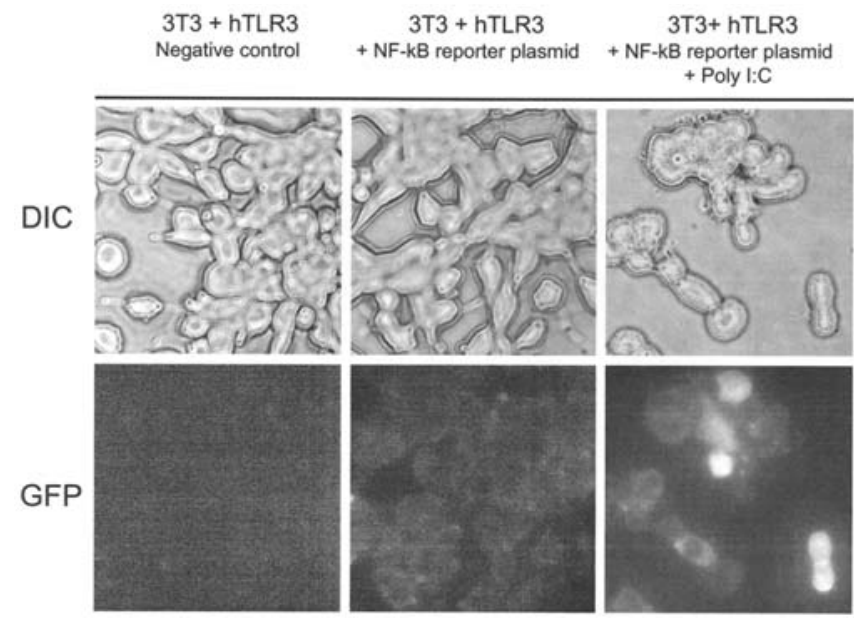

Figure 5. TLR3 stimulation triggers NF- $\mathrm{KB}$ activation. NIH3T3 fibroblasts were simultaneously transfected with TLR3-encoding plasmid pUNOhTLR3 and NF-kB reporter plasmid pNF-kB-hrGFP. Transfected cells were cultivated in the presence and absence of the TLR3 ligand PolyI:C. Fluorescence microscopy was used to analyze NF- $\mathrm{BB}$ activity by the expression levels of green fluorescent protein (GFP) encoded on the reporter plasmid. PolyI:C led to a significantly increased GFP expression, and thus an increased NF- $\mathrm{BB}$ activity, compared to non-stimulated cells. NIH3T3 fibroblasts not harbouring the GFP-encoding $\mathrm{NF}-\kappa \mathrm{B}$ reporter plasmid were used as a negative control.

encoding plasmid pUNO-hTLR3 and NF- $\mathrm{kB}$ reporter plasmid pNF- $\kappa \mathrm{B}-\mathrm{hrGFP}$. Transfected cells were cultivated in the presence and absence of the TLR3 ligand PolyI:C (50 $\mu \mathrm{g} / \mathrm{ml})$. Fluorescence microscopy was used to analyze NF-кB activity by the expression levels of GFP encoded on the reporter plasmid. Fig. 5 shows a significantly increased GFP expression, and thus an increased NF- $\mathrm{KB}$ activity, in response to PolyI:C stimulation of transfected NIH3T3 fibroblasts compared to non-stimulated cells (Fig. 5). NIH3T3 fibroblasts not harbouring the GFP-encoding NF- $\mathrm{BB}$ reporter plasmid were used as a negative control.

\section{Discussion}

TLR3 is directly involved in the regulation of NF-кB. TRAF6-TAK1-TAB2 are recruited to TLR3 upon PolyI:C stimulation and then translocated to the cytosol where TAK1 is phosphorylated. Activation of TAK1 results in the activation

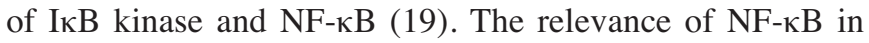
tumor maintenance and tumorigenesis is well accepted and has been observed in a number of human cancers, including breast cancer, non-small-cell lung carcinoma, thyroid cancer and bladder cancer, as well as HNSCC (20-23). Here we showed that the expression of TLR3 in HNSCC is associated with high levels of expression and activity of NF- $\mathrm{B}$. Significant NF- $\kappa$ B levels can already be detected in oral mucosal tissue of heavy smokers or in dysplastic tissue with a markedly further increase during the progression of HNSCC oncogenesis, whereas TLR3 is only significantly expressed in the final stage of head and neck cancer. These data support recent studies indicating that NF- $\mathrm{BB}$ activity is essential for promoting inflammation-associated cancer (24).

It has recently been suggested that TLR3 may directly trigger apoptosis in selected human cancers, such as breast 
cancer (25). Our data indicate that TLR3 participates in the proliferation of HNSCC and thus stress the diversity of human cancers as well as the double-edged character of immunostimulatory approaches in anti-cancer therapies.

The molecular regulation of distinct signal transduction cascades is determined by a variety of parameters such as the amount and species of certain stimuli, individual genetic predispositions, the cytokine profile of the surrounding environment, or the individual characteristics of selected cancers. These data underline the necessity of the individualization of cancer treatment.

Since HNSCC is frequently associated with chronic inflammations, our data strongly suggest that the initial upregulation of NF- $\mathrm{\kappa B}$ in cells of the head and neck region is TLR3 independent and associated rather with the pre-existing chronic inflammation present in smoking individuals. Other types of inflammation, such as acute gingivitis caused by bacterial infiltration, can lead to constitutive upregulation of TLR2 and TLR4 in oral mucosa (26) with the epithelial layer providing the first line of defence against invading pathogens. Thus the expression of TLR3 exclusively in malignant cells is most likely not a remnant of defending persistent viral infections in the oral cavity but an additional mechanism of HNSCC contributing to the constitutive activity of $\mathrm{NF}-\kappa \mathrm{B}$ in cancer cells. Our data have raised a number of questions concerning the molecular mechanisms of the TLR3 function in head and neck cancer and its role within immune escape processes. Studies examining TLR expression on innate leukocytes and other cell types have highlighted the complexity of TLR regulation and the signalling pathways activated in response to various agonists. TLR3 belongs to a subfamily that is localized in the intracellular compartments in late endosomes-lysosomes. There they function to detect pathogen-associated molecular patterns (PAMPs) derived from viral intruders or in case of deficient clearance of apoptotic cells leading to a breakdown of tolerance and autoimmunity (27).

The expression of TLR3 thus reveals a mechanism by which HNSCC could use viral infections, necrotic tissue or the infiltration of immune cells within a human solid tumor to trigger tumor cell growth via a TLR3-dependent activation of NF- $\mathrm{B}$ regulated oncogenes such as c-Myc. Such a mechanism has been described for human embryonic kidney cells where nuclear factor- $\mathrm{\kappa B}$ can be activated via TLR3 in response to treatment with in vitro transcribed mRNA or heterologous RNA released from damaged cells (28).

Future studies will need to further elucidate the cell type specific functions and stimuli of TLR3 in epithelial cancer cells as well as within the network of TLR-expressing cells involved in the innate branch of the immune system.

\section{Acknowledgements}

We thank Stephan Lang, Konrad Sommer and Brigitte Wollmann for the skilful support in some parts of this work. We are grateful to all members of the Department of Otorhinolaryngology for helpful discussions and a comfortable atmosphere. This work was supported by grants from the Mildred-Scheel-Stiftung (Deutsche Krebshilfe), the Wernerand-Klara-Kreitz-Stiftung, the Monika-Kutzner-Stiftung and the Rudolf-Bartling-Stiftung.

\section{References}

1. Sivori S, Falco M, Della Chiesa M, Carlomagno S, Vitale M, Moretta L and Moretta A: $\mathrm{CpG}$ and double-stranded RNA trigger human NK cells by Toll-like receptors: induction of cytokine release and cytotoxicity against tumors and dendritic cells. Proc Natl Acad Sci USA 101: 10116-10121, 2004.

2. Medvedev AE, Kopydlowski KM and Vogel SN: Inhibition of lipopolysaccharide-induced signal transduction in endotoxintolerized mouse macrophages: dysregulation of cytokine, chemokine, and toll-like receptor 2 and 4 gene expression. J Immunol 164: 5564-5574, 2000.

3. Aggarwal S, Takada Y, Singh S, Myers JN and Aggarwal BB: Inhibition of growth and survival of human head and neck squamous cell carcinoma cells by curcumin via modulation of nuclear factor-kappaB signaling. Int J Cancer 111: 679-692, 2004.

4. Rock FL, Hardiman G, Timans JC, Kastelein RA and Bazan JF: A family of human receptors structurally related to Drosophila Toll. Proc Natl Acad Sci USA 95: 588-593, 1998.

5. Dunne A and O'Neill LA: The interleukin-1 receptor/Toll-like receptor superfamily: signal transduction during inflammation and host defense. Sci STKE 2003(171): re3, 2003.

6. Modlin RL: Mammalian toll-like receptors. Ann Allergy Asthma Immunol 88: 543-550, 2002.

7. Pahl HL: Activators and target genes of Rel/NF-kappaB transcription factors. Oncogene 18: 6853-6866, 1999.

8. Luce D, Guenel P, Leclerc A, Brugere J, Point D and Rodriguez J: Alcohol and tobacco consumption in cancer of the mouth, pharynx, and larynx: a study of 316 female patients. Laryngoscope 98: 313-316, 1988.

9. Chin D, Boyle GM, Theile DR, Parsons PG and Coman WB: Molecular introduction to head and neck cancer (HNSCC) carcinogenesis. Br J Plast Surg 57: 595-602, 2004.

10. Douglas WG, Tracy E, Tan D, Yu J, Hicks WL Jr, Rigual NR, Loree TR, Wang Y and Baumann H: Development of head and neck squamous cell carcinoma is associated with altered cytokine responsiveness. Mol Cancer Res 2: 585-593, 2004.

11. Pries R, Thiel A, Brocks C and Wollenberg B: Secretion of tumor-promoting and immune suppressive cytokines by cell lines of head and neck squamous cell carcinoma. In Vivo 20: 45-48, 2006.

12. Pries R and Wollenberg B: Cytokines in head and neck cancer. Cytokine Growth Factor Rev 17: 141-146, 2006.

13. Mann EA, Spiro JD, Chen LL and Kreutzer DL: Cytokine expression by head and neck squamous cell carcinomas. Am J Surg 164: 567-573, 1992.

14. Chen Z, Malhotra PS, Thomas GR, Ondrey FG, Duffey DC, Smith CW, Enamorado I, Yeh NT, Kroog GS, Rudy S, McCullagh L, Mousa S, Quezado M. Herscher LL and Van Waes C: Expression of proinflammatory and proangiogenic cytokines in patients with head and neck cancer. Clin Cancer Res 5: 1369-1379, 1999.

15. Woods KV, El-Naggar A, Clayman GL and Grimm EA: Variable expression of cytokines in human head and neck squamous cell carcinoma cell lines and consistent expression in surgical specimens. Cancer Res 58: 3132-3141, 1998.

16. Giorello L, Clerico L, Pescarolo MP, Vikhanskaya F, Salmona M, Colella G, Bruno S, Mancuso T, Bagnasco L, Russo P and Parodi S: Inhibition of cancer cell growth and c-Myc transcriptional activity by a c-Myc helix 1-type peptide fused to an internalization sequence. Cancer Res 58: 3654-3659, 1998.

17. Hartmann G and Krieg AM: Mechanism and function of a newly identified CpG DNA motif in human primary B cells. J Immunol 164: 944-953, 2000.

18. Kariko K, Bhuyan P, Capodici J and Weissman D: Small interfering RNAs mediate sequence-independent gene suppression and induce immune activation by signaling through toll-like receptor 3. J Immunol 172: 6545-6549, 2004.

19. Jiang Z, Mak TW, Sen G and Li X: Toll-like receptor 3mediated activation of NF-kappaB and IRF3 diverges at TollIL-1 receptor domain-containing adapter inducing IFN-beta. Proc Natl Acad Sci USA 101: 3533-3538, 2004.

20. Seya T, Akazawa T, Uehori J, Matsumoto M, Azuma I and Toyoshima K: Role of toll-like receptors and their adaptors in adjuvant immunotherapy for cancer. Anticancer Res 23: 4369-4376, 2003.

21. Bharti AC and Aggarwal BB: Nuclear factor-kappa B and cancer: its role in prevention and therapy. Biochem Pharmacol 64: 883-888, 2002. 
22. Rath PC and Aggarwal BB: Antiproliferative effects of IFNalpha correlate with the downregulation of nuclear factor-kappa B in human Burkitt lymphoma Daudi cells. J Interferon Cytokine Res 21: 523-528, 2001.

23. Pikarsky E, Porat RM, Stein I, Abramovitch R, Amit S, Kasem S, Gutkovich-Pyest E, Urieli-Shoval S, Galun E and Ben-Neriah Y: NF-kappaB functions as a tumour promoter in inflammationassociated cancer. Nature 431: 461-466, 2004.

24. Greten FR and Karin M: The IKK/NF-kappaB activation pathway-a target for prevention and treatment of cancer. Cancer Lett 206: 193-199, 2004.
25. Salaun B, Coste I, Rissoan MC, Lebecque SJ and Renno T: TLR3 can directly trigger apoptosis in human cancer cells. J Immunol 176: 4894-4901, 2006.

26. Wang PL, Ohura K, Fujii T, Oido-Mori M, Kowashi Y, Kikuchi M, Suetsugu Y and Tanaka J: DNA microarray analysis of human gingival fibroblasts from healthy and inflammatory gingival tissues. Biochem Biophys Res Commun 305: 970-973, 2003.

27. Iwasaki A and Medzhitov R: Toll-like receptor control of the adaptive immune responses. Nat Immunol 5: 987-995, 2004.

28. Kariko K, Ni H, Capodici J, Lamphier M and Weissman D: mRNA is an endogenous ligand for Toll-like receptor 3. J Biol Chem 279: 12542-12550, 2004. 